\title{
Utilizing Electronic Medical Records to Standardize Handoffs in Academic Ophthalmology
}

\author{
Lynn W. Sun, MD, PhD ${ }^{1}$ Andrea L. Stahulak, MD ${ }^{1}$ Deborah M. Costakos, MD, MS ${ }^{1}$ \\ ${ }^{1}$ Department of Ophthalmology, Medical College of Wisconsin, \\ Milwaukee, Wisconsin \\ J Acad Ophthalmol 2020;12:e205-e213. \\ Address for correspondence Deborah M. Costakos, MD, MS, \\ Department of Ophthalmology, Eye Institute, Medical College of \\ Wisconsin, 925 North, 87th Street, Milwaukee, WI 53226 \\ (e-mail: dcostakos@mcw.edu).
}

\begin{abstract}
Keywords

- handoff

- handover

- electronic medical record

Purpose Formalized handoff procedures have been shown to increase patient safety and quality of care across multiple medical and surgical specialties, ${ }^{1-4}$ but literature regarding handoffs in ophthalmology remains sparse. We instituted a standardized handoff utilizing an electronic medical record (EMR) system to improve care for patients shared by multiple resident physicians across weekday, weeknight, and weekend duty shifts. We measured efficiency, efficacy, and resident satisfaction before and after the standardized handoff was implemented.

Methods Resident physicians surveyed were primarily responsible for patient care on consult and call services at two quaternary academic medical centers in a major metropolitan area. Patient care was performed in outpatient, emergency, and inpatient settings. Annual anonymous questionnaires consisting of 6 questions were used to collect pre- and postintervention impressions of the standardized EMR handoff process from ophthalmology resident physicians ( 9 per year; 3 preintervention years and 1 postintervention year). An additional anonymous postintervention questionnaire consisting of 12 questions was used to further characterize resident response to the newly implemented handoff procedure.

Results Prior to implementation of a standardized EMR-based handoff procedure, residents unanimously reported incomplete, infrequently updated handoff reports that did not include important clinical and/or psychosocial information. Following implementation, residents reported a statistically significant increase in completeness and timeliness of handoff reports. Additionally, resident perception of EMR handoff utility, efficiency, and usability were comprehensively favorable. Residents reported handoffs only added a mean of 6.5 minutes to a typical duty shift.

Conclusion Implementation of our protocol dramatically improved resident perceptions of the handoff process at our institution. Improvements included increased quality, ease-of-use, and efficiency. Our standardized EMR-based handoff procedure may be of use to other ambulatory-based services.
\end{abstract}

Handoff, the process of transferring patients from outgoing providers to incoming providers at the end of a duty shift, has been well established as a major independent risk factor for medical errors. ${ }^{1,2}$ Much effort has been directed toward reduction of medical errors associated with handoffs in inpa- tient services such as internal medicine, pediatrics, and general surgery. ${ }^{1-4}$ In 2009 a Handoff Task Force dedicated to the standardization of handoffs in internal medicine hospitalist services set forth a series of evidence-based recommendations, ${ }^{5}$ reprinted here with permission of the authors: received

August 3, 2020

accepted

August 31, 2020
DOI https://doi.org/

10.1055/s-0040-1718566. ISSN 2475-4757.
Copyright $\odot 2020$ by Thieme Medical Publishers, Inc., 333 Seventh Avenue, New York, NY 10001, USA. Tel: +1(212) 760-0888.
License terms

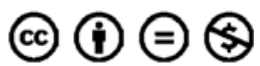




\section{Hospitalist Handoffs: Task Force Recommendations}

A formally recognized handoff plan should be instituted at the end of a shift or change in service (Class I Level of evidence C).

\section{Program Recommendations}

Hospitalist programs should specify the following for those clinicians engaged in handoffs:

1. Time during shift dedicated for verbal exchange of information (Class I Level of evidence C).

2. Template OR technology solution to be used for accessing and recording patient information ["signout" or "patient list"] during handoff (Class I Level of evidence B).

3. Training for new users on handoff expectations (Class I Level of evidence $C$ ).

4. Tracking system to document the correct hospitalist caring for a specific patient after a service change (Class I Level of evidence C).

\section{Verbal Exchange Recommendations}

Handoffs should include a verbal exchange of patient information that is characterized by the following:

- Interactive process is used during the verbal exchange (Class I Level of evidence C).

- Ill patients are given priority during the verbal exchange (Class I Level of evidence C).

- Insight on what to anticipate or what to do is the focus of the verbal exchange (Class I Level of evidence C).

\section{Content Exchange Recommendations}

Handoffs should use a context exchange summary (e.g., signout or patient list) which is characterized by the following:

- All patients that are handed off are included (Class I Level of evidence $\mathrm{C}$ ).

- Available in a centralized location (Class I Level of evidence $\mathrm{C}$ ).

- All data kept up-to-date (Class I Level of evidence C).

- Anticipated events for incoming hospitalist are clearly labeled (Class I Level of evidence $C$ ).

- Action items for incoming hospitalist are highlighted (i.e., To-do) (Class I Level of evidence C).

Based on these recommendations, standardized handoff protocols such as I-PASS have been proposed and implemented. ${ }^{6,7}$ Recent studies across numerous specialties have convincingly demonstrated positive outcomes associated with adoption of such protocols in patient safety, ${ }^{8-11}$ in resident efficiency and satisfaction, ${ }^{6,12-15}$ and in both. ${ }^{16-19}$

In contrast, little research has been done regarding standardization of handoff procedures in our field and no systematic guidelines have been published to our knowledge. This perhaps owes to ophthalmology being primarily an outpatient-based specialty. Nonetheless, ophthalmology services frequently care for patients requiring care across two or more duty shifts and are vulnerable to the same risks for medical error associated with handoffs in other specialties. At present, there is little published evidence of standardized processes for handoffs in ophthalmology, potentially indicating a deficiency that may compromise efficiency and safety of patient care. In addition, ophthalmology services often cover several hospitals and outpatient clinics, which precludes faceto-face handoffs at fixed times and in centralized locations. With the widespread adoption of electronic medical records (EMRs), we believe these popular tools can be used as an integral component of a standardized handoff procedure, and that the Handoff Task Force recommendations can be adapted to maximize the benefit of technology.

In this project, we propose and implement a modified standardized handoff procedure loosely based on the Arora et al recommendations listed above ${ }^{5}$ and the I-PASS protocol. ${ }^{7}$ A centralized EMR accessible by all resident, fellow, and faculty physicians was used to track patients and facilitate transfer of critical information between duty shifts at our institution. Handoff information provided in the EMR handoff template included brief history of present illnesses/summaries, ongoing clinical care, anticipated events and responses, and action items. Following implementation of this system, we assessed whether standardizing handoffs using the EMR resulted in safer and more efficient patient care in ophthalmology. Preand postinterventional resident physician perceptions of the handoff procedure and their ability to safely and efficiently deliver care were measured using an anonymous questionnaire tool. Similar pre-/post-studies have been done in internal medicine and pediatrics. $6,9,12,13,15,17-22$ We also highlight use of EMRs in a standardized handoff procedure in ophthalmology, which may serve as a model for other programs and contribute to a nascent discussion of utility and necessity of standardized handoffs in primarily outpatient specialties.

\section{Methods}

\section{Study Group and Duty Shifts}

This study was conducted at two quaternary academic medical centers (585-bed and 306-bed) in a major metropolitan area with a population of 1.57 million. The study was ruled exempt from approval by the institutional review board and adheres to the principles set forth by the Declaration of Helsinki. Resident physicians surveyed were primarily responsible for patient care on consult and call services. The preintervention group comprised of 9 residents a year surveyed in November of 2015, 2016, and 2017 prior to implementation of a standardized EMR-based handoff procedure in August of 2018. The postintervention group comprised of 9 residents surveyed in November of 2018 and May of 2019 following implementation. Patients being handed off included:

- Long-term inpatients requiring follow-up or interventions across multiple duty shifts.

- New patients arriving during overnight duty shifts requiring further care during daytime duty shifts.

- Outpatients requiring further care, whether in outpatient, emergency, or inpatient settings.

- Patients passed from fellow and attending physicians to the resident service, including expected patients that had 
not yet arrived in the clinic, the emergency department, or the inpatient floors.

Handoff times occurred twice a day from Monday to Friday, once at 7 a.m. and once at 4:30 p.m. Each weekday duty shift was staffed by the same resident physician for a given calendar month (the "consult resident"), while each weeknight duty shift was staffed by a different resident each night (the "call resident"). On Saturday and Sunday, handoffs occurred at 4:30 p.m. each day; one call resident staffed Friday 4:30 p.m. to Saturday 4:30 p.m., as well as Sunday 4:30 p.m. to Monday 7 a.m. A different call resident staffed Saturday 4:30 p.m. to Sunday 4:30 p.m. At the end of each calendar month, an extra handoff occurred from one consult resident to the next.

\section{Handoff Procedures}

Preintervention handoffs lacked standardization and were highly variable. Handoffs could include information communicated by email, text page, EMR messaging, or face-to-face discussion. There was no reliable, centralized EMR-based master list of all patients being followed by the ophthalmology service.

After implementation of the new handoff procedure in August of 2018, the protocol detailed in - Fig. 1 was followed.

\section{Evaluation of Pre-|Postinterventional Resident Perceptions of Handoff Procedures}

An anonymous, standardized, annual questionnaire distributed by the Medical College of Wisconsin (Questionnaire 1) was used to assess resident experience before and after the implementation of a standardized handoff procedure. The questionnaire comprised of five Yes/No items and a sixth question was graded on frequency $(1$, never; 2 , rarely; 3 , sometimes; 4 , most of the time; 5 , always):

Questionnaire 1

1. Does your program have a standardized handoff procedure?

2. Handoff reports include all patients.

\title{
Standardized Ambulatory Service Handoff Protocol
}

\author{
1. All patients being handed off are added to a \\ centralized Handoff List on the Electronic \\ Medical Record (EMR).
}

\section{A brief history of present illness (HPI) is provided for each patient in the list.}

3. Any anticipated events are detailed.

4. Any action items are explicitly stated.

5. The Handoff List is updated by the outgoing resident at end of each duty shift.

6. The oncoming resident checks the Handoff List at the beginning of each duty shift and as needed thereafter.

\section{The outgoing resident remains available for questions by phone for 1 hour after duty shift.}

Fig. 1 Recommended standardized handoff protocol for ambulatory services. This figure presents the handoff protocol used as the intervention in this study. It was implemented on our primarily ambulatory ophthalmology service in August of 2018. 
3. Handoff reports are updated daily.

4. Handoff reports include clinical information important to medical decision making.

5. Handoff reports include psychosocial factors that may become important.

6. How often is the standardized handoff process utilized?

Of note, residents were able to abstain from answering, and therefore not every resident responded to every question. Thus, $N$ varied depending on both year and question. This questionnaire was distributed annually in November 2015 to 2017 prior to intervention. The postintervention questionnaire was distributed in November of 2018.

An additional questionnaire (Questionnaire 2) was distributed in May of 2019 to better characterize postintervention resident perceptions of the handoff process. This questionnaire consisted 11 questions graded on frequency ( 1 , never; 2 , rarely; 3, sometimes; 4, most of the time; 5 , always). Generally, responses of "Sometimes," "Most of the time," or "Always" were considered favorable. On Question 8, responses of "Never" or "Rarely" were considered favorable. Additionally, a twelfth question was graded on time in minutes:

Questionnaire 2

1. EMR handoff includes all patients.

2. EMR handoff is updated daily.

3. EMR is utilized at each shift change.

4. EMR handoff includes clinical information important to medical decision making.

5. EMR handoff contains an up-to-date "to do" list on each patient.

6. EMR handoff includes important psychosocial information.

7. I am confident that I have the information I need to take care of patients handed off to me.

8. I need to contact the outgoing resident for more information when using the EMR handoff system.

9. EMR handoff is easy to use.

10. EMR handoff improves my overall efficiency.

11. I am satisfied with the EMR standardized handoff system.

12. How much time do you need to complete the standardized handoff for the incoming resident?

Once again, although all 9 residents completed the questionnaire, residents were allowed to abstain from answering. Therefore, $N$ varied depending on question. Questions 2, 3, 5, and 7 in Questionnaire 2 were written to explicitly match questions 2, 3, 4, and 5 in Questionnaire 1 . When comparing trends, answers ranging from 3 (Sometimes) to 5 (Always) in Questionnaire 2 were considered equivalent to a "Yes" response in Questionnaire 1. However, answers obtained from Questionnaire 2 were not used in statistical analysis.

\section{Statistical Analysis}

For each Yes/No question in Questionnaire 1, preintervention responses from 2015, 2016, and 2017 and conglomerate data from 2015 to 2017 were compared against postintervention responses from 2018 using two-tailed Pearson's chi-square tests. In 2016, several questions had an $N=1$. For these questions, a Fisher's exact test was used to assess statistical significance.

Although responses from Questionnaire 2 were processed into Yes/No responses (see above) for purposes of comparison against Questionnaire 1, no statistical analyses were conducted on this data. Likewise, no statistical analyses were conducted on nonbinary questions (i.e., those graded on frequency or time required).

\section{Results}

\section{Demographics}

Standardized EMR-based handoff procedures detailed in - Fig. 1 were implemented in the ophthalmology service in August 2018. Each year, 9 resident physicians primarily responsible for all consult and call services at our quaternary-level medical centers were surveyed in November. Residents had the option of abstaining from answering any question on the questionnaires. Some questions were not presented if a preceding question was answered no. Resultantly, $N$ varied by question and by year. Preintervention data was collected using Questionnaire 1 (see "Methods") in November of 2015, 2016, and 2017. In 2015 and 2016, all 9 residents answered at least one handoff-related question. In 2017, 7 residents answered at least one handoff-related question. Postintervention data was collected using Questionnaire 1 in November 2018, 3 months after intervention, and Questionnaire 2 in May 2019, 9 months after intervention. In 2018, 6 residents answered at least one handoffrelated question. In 2019, 9 residents answered at least one handoff-related question.

\section{Resident Perceptions of the Handoff Process Pre- and Postintervention}

Questionnaire 1 responses pre- and postintervention are summarized in -Table 1 and graphed in -Fig. 2 (data from 2015 to 2018) and -Fig. 3 (data from 2015 to 2019; 2019 data abstracted from Questionnaire 2, below).

Prior to implementation of the standardized EMR-based handoff procedure, a minority of residents completing Questionnaire 1 in 2015, 2016, and 2017 reported using any type of standardized handoff procedure on the ophthalmology service ( 7 of 25 respondents, or $28 \%$ over 2015-2017). Of those who did use a standardized handoff procedure, $82 \%$ (9 of 11 respondents) reported using the procedure frequently (defined as an answer of "Sometimes," "Most of the time," or "Always"), while $18 \%$ ( 2 of 11 ) reported using the procedure infrequently (defined as "Rarely" or "Never"). However, these residents also stated handoffs did not include all patients, were not updated daily, did not include clinical information important to medical decision making, and did not include psychosocial factors that may become important in patient care.

Three months after implementation of the standardized EMR-based handoff procedure, a majority of residents completing Questionnaire 1 in 2018 reported a standardized handoff procedure (6 of 7 respondents, or $86 \%$ in 2018). This represents a statistically significant increase as compared with $2015(p=0.04), 2016(p=0.004)$, and conglomerate 
Table 1 Pre- and postintervention comparison of handoff procedures

\begin{tabular}{|l|l|l|l|l|l|l|}
\hline & 2015 & 2016 & 2017 & $2015-2017$ & 2018 & 2019 \\
\hline 1. Program has a standardized handover procedure & $33 \%(9)$ & $11 \%(9)$ & $43 \%(9)$ & $28 \%(25)$ & $86 \%(7)$ & N/A \\
\hline 2. Written reports include all patients & $0 \%(3)$ & $0 \%(1)$ & $0 \%(7)$ & $0 \%(11)$ & $66 \%(6)$ & $100 \%(7)$ \\
\hline 3. Written reports are updated daily & $0 \%(3)$ & $0 \%(1)$ & $0 \%(7)$ & $0 \%(11)$ & $50 \%(6)$ & $86 \%(7)$ \\
\hline $\begin{array}{l}\text { 4. Written reports include clinical information } \\
\text { important to medical decision making }\end{array}$ & $0 \%(3)$ & $0 \%(1)$ & $0 \%(7)$ & $0 \%(11)$ & $66 \%(6)$ & $86 \%(7)$ \\
\hline $\begin{array}{l}\text { 5. Written reports include psychosocial factors } \\
\text { that may become important }\end{array}$ & $0 \%(3)$ & $0 \%(1)$ & $0 \%(7)$ & $0 \%(11)$ & $33 \%(6)$ & $71 \%(7)$ \\
\hline
\end{tabular}

Note: Table includes data from Questionnaire 1 (2015-2018) and Questionnaire 2 (2019). Implementation of standardized handoff procedure occurred between 2017 and 2018 (vertical line). Data from Questionnaire 2 was converted from frequency-based responses (see "Methods"), and is presented in column 7 in italics. Questionnaire questions are listed in column 1; columns 2-7 present percentage of "Yes" responses ( $N$ in parentheses).
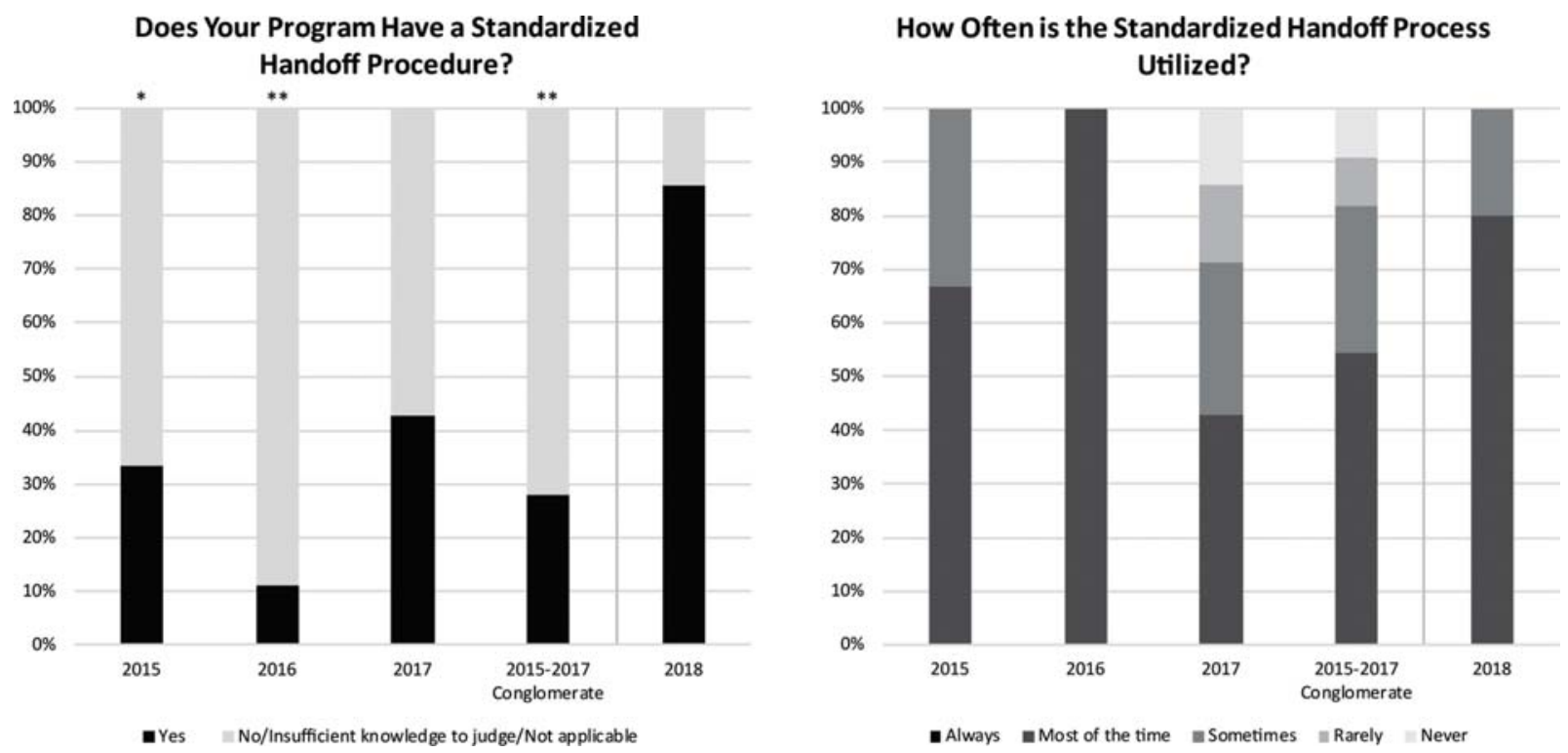

Fig. 2 Handoff procedure trends from 2015 to 2018. Data in this figure is derived from Questionnaire 1 and represent responses assessing overall handoff procedural trends. Relevant questions are listed at the top of each graph. Preintervention data are depicted left of the gray line. ${ }^{*} p<0.05 ;{ }^{* *} p<0.005$.

data from 2015 to 2017 ( $p=0.007$ ). Of these residents, $83 \%$ ( 5 of 6 ) now reported using the procedure frequently, while $17 \%$ ( 1 of 6 ) abstained from reporting frequency of use. A majority of these residents also reported handoffs included all patients ( 4 of 6 , or $66 \%$ ), a statistically significant increase as compared with $2017(p=0.01)$ and conglomerate data from 2015 to $2017(p=0.003)$. Half of these residents additionally reported handoffs were now updated daily (3 of 6 , or $50 \%$ ), a statistically significant increase as compared with $2017(p=0.04)$ and conglomerate data from 2015 to $2017(p=0.01)$. Finally, residents stated handoffs now included clinical information important to medical decision making ( 4 of 6 , or $66 \%$ ), a statistically significant increase as compared with $2017(p=0.01)$ and conglomerate data from 2015 to 2017 ( $p=0.003)$. In 2018, a minority $(33 \%)$ of residents reported handoffs included psychosocial factors that may become important to patient care.

In 2019, residents completing Questionnaire 2 unanimously reported handoffs now included all patients. A larger majority of residents as compared with 2018 now reported handoffs were updated daily (6 of 7 or $86 \%$ ) and included clinical information important to medical decision making (6 of 7 or $86 \%$ ). A majority of residents ( 5 of 7 or $71 \%$ ) also reported handoffs included psychosocial factors that may become important to patient care. Because Questionnaire 2 consisted of frequency-based responses, answers of "Sometimes," "Most of the time," and "Always" were considered equivalent to "Yes" for purposes of comparison to Questionnaire 1. Answers of "Rarely" and "Never" were considered equivalent to "No."

\section{Characterization of Resident Perceptions of Handoffs Postintervention}

Questionnaire 2 responses postintervention are summarized in - Table 2 and graphed in - Fig. 4. Nine months following implementation of a standardized EMR-based handoff procedure, resident perceptions were further characterized using a frequency-based questionnaire. Generally, responses of "Sometimes" or better were considered favorable; on one question assessing the frequency with which the outgoing 

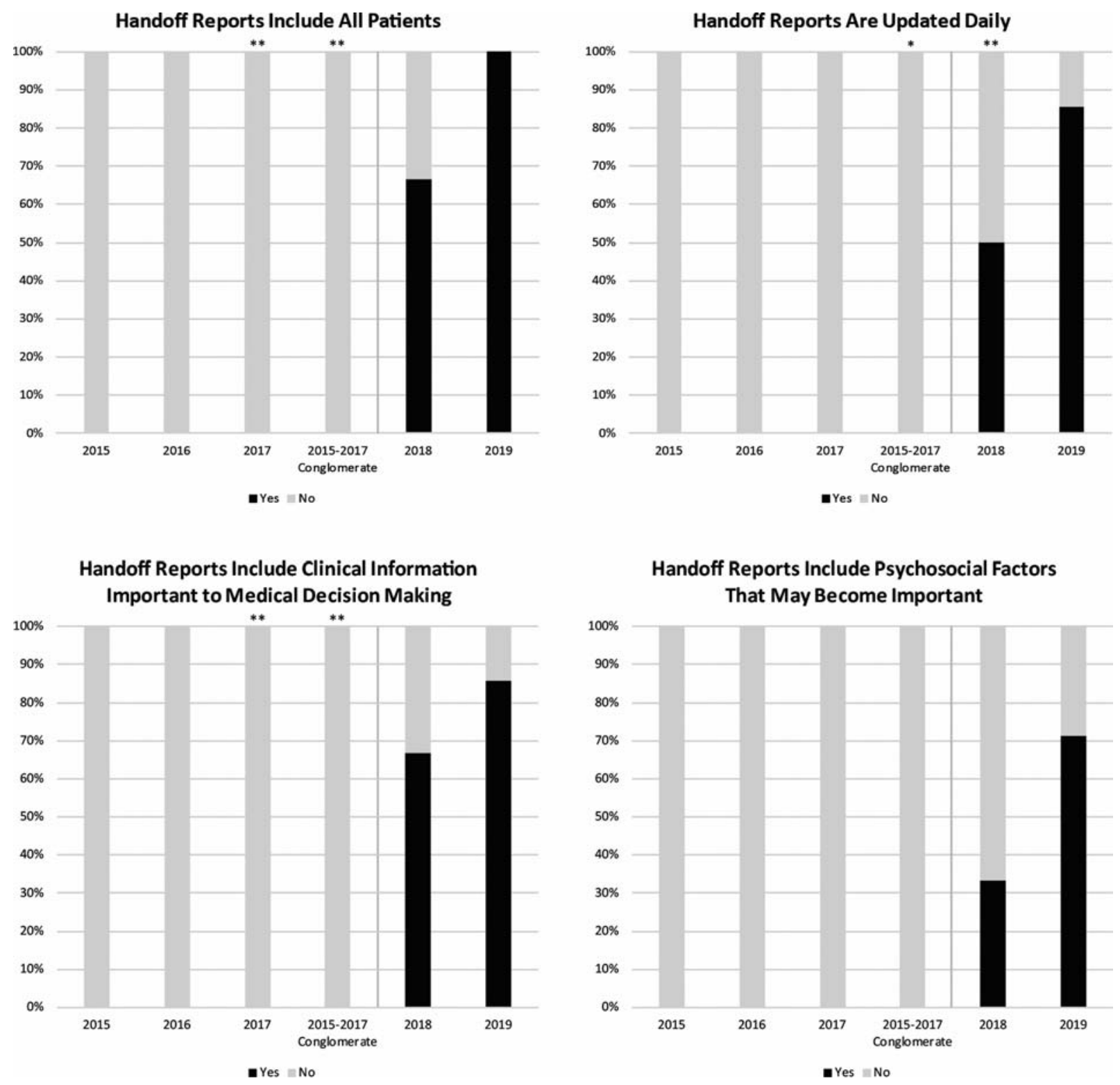

Fig. 3 Handoff content and utility trends from 2015 to 2019. Data in this figure is derived from Questionnaire 1 and 2 and represent assessment of content and utility of handoffs pre- and postintervention. Relevant questions are listed at the top of each graph. 2019 data was collected as frequency-based responses in Questionnaire 2 and abstracted to Yes/No responses. Preintervention data are depicted left of the gray line. For statistical purposes, only 2018 data was considered postinterventional. ${ }^{*} p<0.05 ;{ }^{* *} p<0.005$.

resident needed to be contacted for more information, responses of "Rarely" or "Never" were considered favorable.

Six questions addressed completeness and usefulness of EMR handoff reports. All respondents reported EMR handoffs included all patients either "Most of the time" or "Always." Six of 7 (86\%) of residents reported EMR handoffs were updated every day "Sometimes" or "Most of the time," though 1 of 7 or $14 \%$ stated EMR handoffs were only "Rarely" updated daily. EMR handoffs were reported to be used at each shift change "Sometimes" or "Most of the time" by 6 of 7 respondents ( $86 \%$ ), though 1 of 7 (14\%) stated EMR handoffs were only "Rarely" used at each shift change. Clinical information important to medical decision making was reported "Sometimes," "Most of the time," or "Always" by 6 of 7 residents (28\%), though 1 of 7 or $14 \%$ reported this was only "Rarely" the case. All residents reported EMR handoffs included updated action items "Most of the time." Most residents (5 of 7 or $71 \%$ ) reported EMR handoffs "Sometimes" contained important psychosocial information, while 2 of 7 or $28 \%$ reported EMR handoffs only "Rarely" contained psychosocial details.

Five questions addressed efficiency and usability of EMR handoff reports. Residents unanimously reported high confidence in having information needed for patient care ( 6 of 7 or $86 \%$ "Most of the time," 1 of 7 or $14 \%$ "Always"). Three of 7 (43\%) residents reported contacting the outgoing resident only "Rarely"; 4 of 7 (71\%) residents reported needing to contact the outgoing resident more frequently ("Sometimes," "Most of the time," or "Always"). All residents reported good ease-of-use "Most of the time" or "Always." All residents also reported improved efficiency "Sometimes," "Most of the time," or 
Table 2 Postintervention resident perceptions of handoff procedures

\begin{tabular}{|c|c|c|c|c|c|}
\hline & $\begin{array}{l}1- \\
\text { Never }\end{array}$ & $\begin{array}{l}2- \\
\text { Rarely }\end{array}$ & $\begin{array}{l}\text { 3- } \\
\text { Sometimes }\end{array}$ & $\begin{array}{l}\text { 4-Most } \\
\text { of the } \\
\text { time }\end{array}$ & 5-Always \\
\hline 1. The EMR handoff includes all patients & $0 \%$ & $0 \%$ & $0 \%$ & $86 \%$ & $14 \%$ \\
\hline 2. The EMR handoff is updated daily & $0 \%$ & $14 \%$ & $57 \%$ & $29 \%$ & $0 \%$ \\
\hline 3. The EMR handoff is utilized at each shift change & $0 \%$ & $14 \%$ & $29 \%$ & $57 \%$ & $0 \%$ \\
\hline $\begin{array}{l}\text { 4. The EMR handoff includes clinical information important } \\
\text { to medical decision making }\end{array}$ & $0 \%$ & $14 \%$ & $14 \%$ & $43 \%$ & $29 \%$ \\
\hline 5. The EMR handoff contains an up-to-date "to do" list on each patient & $0 \%$ & $0 \%$ & $0 \%$ & $100 \%$ & $0 \%$ \\
\hline 6. The EMR handoff includes important psychosocial information & $0 \%$ & $29 \%$ & $71 \%$ & $0 \%$ & $0 \%$ \\
\hline $\begin{array}{l}\text { 7. I am confident that I have the information I need to take care } \\
\text { of patients handed off to me }\end{array}$ & $0 \%$ & $0 \%$ & $0 \%$ & $86 \%$ & $14 \%$ \\
\hline $\begin{array}{l}\text { 8. I need to contact the outgoing resident for more information } \\
\text { when using the EMR handoff system }\end{array}$ & $0 \%$ & $43 \%$ & $14 \%$ & $29 \%$ & $14 \%$ \\
\hline 9. The EMR handoff is easy to use & $0 \%$ & $0 \%$ & $0 \%$ & $29 \%$ & $71 \%$ \\
\hline 10. The EMR handoff improves my overall efficiency & $0 \%$ & $0 \%$ & $57 \%$ & $14 \%$ & $29 \%$ \\
\hline 11. I am satisfied with the EMR handoff system & $0 \%$ & $0 \%$ & $29 \%$ & $29 \%$ & $43 \%$ \\
\hline
\end{tabular}

Abbreviation: EMR, electronic medical record.

Note: Table includes data from Questionnaire 2 (2019, postintervention). Questionnaire questions are listed in column 1. Responses are presented as percentage of total responding to listed frequencies. $N=7$ for all responses.

"Always." Likewise, all residents reported satisfaction with the standardized EMR-based handoff system "Sometimes," "Most of the time," or "Always."

We also assessed estimated amount of time needed by the outgoing resident to complete handoffs. Seven residents responded to this question. In some cases, responses were given as a range (e.g., 2-5); in these cases, the midpoint of the given range (e.g., 3.5) was used in calculating overall mean time needed. Respondents reported requiring a mean of 6.5 minutes and a median of 5 minutes to complete the EMR-based handoff report (standard deviation $=4.9$ minutes). The range of time required was 2 to 15 minutes.

\section{Discussion}

Prior to implementation of standardized handoffs, a majority of residents did not report usage of any standardized handoff procedure. Those who did reported standardized handoffs nonetheless unanimously reported incomplete, infrequently updated handoff reports that did not include important clinical and/or psychosocial information. Preintervention handoffs did not use centralized, written handoff reports or procedures conforming to hospitalist recommendations. ${ }^{5}$

Following implementation, residents reported increased completeness and usefulness of EMR-based handoffs. Our department noted a statistically significant increase in the proportion of residents recognizing standardized handoff procedures. We also saw a statistically significant increase in the number of residents reporting daily updates to written reports that include all patients and include clinical information important to medical decision-making. A majority of residents now report that standardized handoffs are occurring at the end of most shifts. Residents also responded favorably regarding the comprehensiveness, utility, and timeliness of handoff reports in the EMR. Where a comparison could be made, favorable responses appeared to increase between 3 and 9 months postintervention, suggesting increased satisfaction as residents gain familiarity with the procedure.

Residents also reported satisfaction with the efficiency and usability of postintervention handoffs. Respondents reported excellent ease-of-use, increased efficiency, high confidence, and overall satisfaction with the EMR-based standardized handoff procedure. It is important to note the newly implemented handoff procedure did not appear to add an undue burden to consulting and on-call residents, with residents reporting a mean/median of around 5 to 6 minutes spent on handoffs.

Limitations of this study primarily stem from the small sample size. This is due in part to the small class size of most ophthalmology residencies, with our program recruiting only three residents a year. Residents are also permitted to abstain from answering questions due to personal preference or because they do not feel qualified to answer certain questions. Consequently, our $N$ is low in most cases. Further conglomeration of data would likely lead to more statistically significant results, and we look forward to monitoring the responses from upcoming annual surveys.

Implementation of a standardized EMR-based handoff procedure resulted in a comprehensive improvement in quality, efficacy, and efficiency of handoffs in our quaternary hospitalbased ophthalmology service. We believe these results may be generalizable to most if not all ophthalmology services that require handoffs between different duty shifts, as well as to other primarily ambulatory services that nonetheless treat patients in a variety of care contexts. In this article, we present our EMR handoff protocol as a prototype for other services 

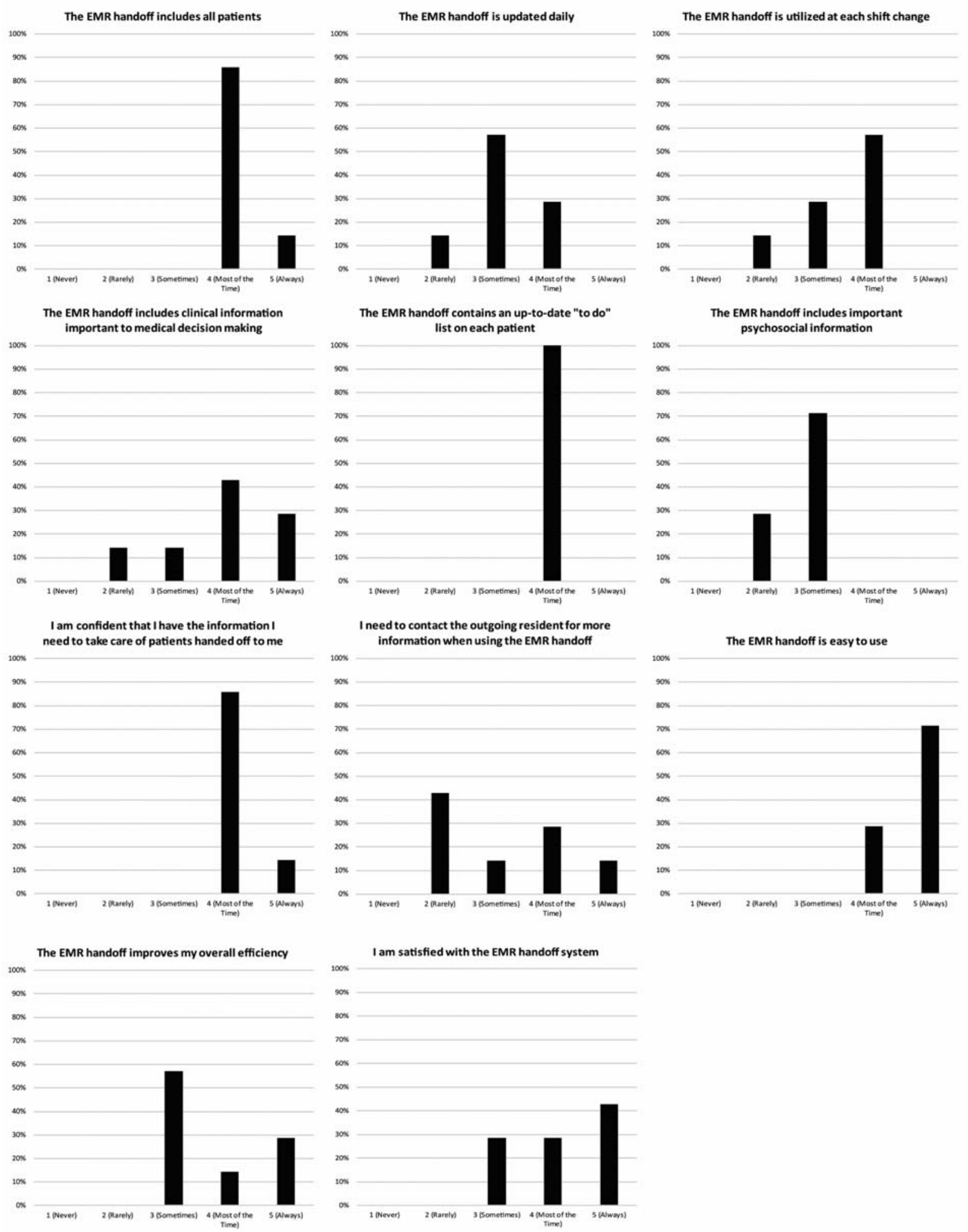

Fig. 4 Resident perception of handoffs, 2019. Data in this figure is derived from Questionnaire 2 and represent resident assessment of utility, content, efficiency, and usability of electronic medical record (EMR)-based handoffs. Relevant questions are listed at the top of each graph. All data are postinterventional.

with similar characteristics and needs. We hope to elicit a dialogue within ophthalmology and other outpatient-based specialties regarding the necessity and utility of handoffs in increasing the efficiency, efficacy, and quality of care for our patients.
Funding

None.

Conflict of Interest

None declared. 


\section{References}

1 Arora V, Johnson J, Lovinger D, Humphrey HJ, Meltzer DO. Communication failures in patient sign-out and suggestions for improvement: a critical incident analysis. Qual Saf Health Care 2005;14(06):401-407

2 Horwitz LI, Moin T, Krumholz HM, Wang L, Bradley EH. Consequences of inadequate sign-out for patient care. Arch Intern Med 2008;168(16):1755-1760

3 Solet DJ, Norvell JM, Rutan GH, Frankel RM. Lost in translation: challenges and opportunities in physician-to-physician communication during patient handoffs. Acad Med 2005;80(12): 1094-1099

4 Wray CM, Chaudhry S, Pincavage A, et al. Resident shift handoff strategies in US internal medicine residency programs. JAMA 2016;316(21):2273-2275

5 Arora VM, Manjarrez E, Dressler DD, Basaviah P, Halasyamani L, Kripalani S. Hospitalist handoffs: a systematic review and task force recommendations. J Hosp Med 2009;4(07):433-440

6 Starmer AJ, O'Toole JK, Rosenbluth GI-PASS Study Education Executive Committee. , et al; . Development, implementation, and dissemination of the I-PASS handoff curriculum: a multisite educational intervention to improve patient handoffs. Acad Med 2014;89(06):876-884

7 Starmer AJ, Spector ND, Srivastava R, Allen AD, Landrigan CP, Sectish TCI-PASS Study Group. I-pass, a mnemonic to standardize verbal handoffs. Pediatrics 2012;129(02):201-204

8 Arora VM, Berhie S, Horwitz LI, Saathoff M, Staisiunas P, Farnan JM. Using standardized videos to validate a measure of handoff quality: the handoff mini-clinical examination exercise. J Hosp Med 2014;9(07):441-446

9 Doers ME, Beniwal-Patel P, Kuester J, Fletcher KE. Feedback to achieve improved sign-out technique. Am J Med Qual 2015;30 (04):353-358

10 Feraco AM, Starmer AJ, Sectish TC, Spector ND, West DC, Landrigan $\mathrm{CP}$. Reliability of verbal handoff assessment and handoff quality before and after implementation of a resident handoff bundle. Acad Pediatr 2016;16(06):524-531

11 Lee SH, Fisher DA, Mah H, Goh WP, Phan PH. A qualitative study of sign-out processes between primary and on-call residents: rela- tionships in information exchange, responsibility and accountability. Int J Qual Health Care 2017;29(05):646-653

12 Starmer AJ, Spector ND, Srivastava RI-PASS Study Group. , et al; . Changes in medical errors after implementation of a handoff program. N Engl J Med 2014;371(19):1803-1812

13 Allen S, Caton C, Cluver J, Mainous AG III, Clyburn B. Targeting improvements in patient safety at a large academic center: an institutional handoff curriculum for graduate medical education. Acad Med 2014;89(10):1366-1369

14 Coffey M, Thomson K, Li SA, et al. Resident experiences with implementation of the I-PASS handoff bundle. J Grad Med Educ 2017;9(03):313-320

15 Sawatsky AP, Mikhael JR, Punatar AD, Nassar AA, Agrwal N. The effects of deliberate practice and feedback to teach standardized handoff communication on the knowledge, attitudes, and practices of first-year residents. Teach Learn Med 2013;25(04):279-284

16 Arora VM, Greenstein EA, Woodruff JN, Staisiunas PG, Farnan JM. Implementing peer evaluation of handoffs: associations with experience and workload. J Hosp Med 2013;8(03):132-136

17 McCrory MC, Aboumatar H, Custer JW, Yang CP, Hunt EA. "ABCSBAR" training improves simulated critical patient hand-off by pediatric interns. Pediatr Emerg Care 2012;28(06):538-543

18 Aboumatar H, Allison RD, Feldman L, Woods K, Thomas P, Wiener C. Focus on transitions of care: description and evaluation of an educational intervention for internal medicine residents. Am J Med Qual 2014;29(06):522-529

19 Johnson DP, Zimmerman K, Staples B, McGann KA, Frush K, Turner DA. Multicenter development, implementation, and patient safety impacts of a simulation-based module to teach handovers to pediatric residents. Hosp Pediatr 2015;5(03):154-159

20 Farnan JM, Paro JA, Rodriguez RM, et al. Hand-off education and evaluation: piloting the observed simulated hand-off experience (OSHE). J Gen Intern Med 2010;25(02):129-134

21 Gakhar B, Spencer AL. Using direct observation, formal evaluation, and an interactive curriculum to improve the sign-out practices of internal medicine interns. Acad Med 2010;85(07):1182-1188

22 Rüdiger-Stürchler M, Keller DI, Bingisser R. Emergency physician intershift handover - can a dINAMO checklist speed it up and improve quality? Swiss Med Wkly 2010;140:w13085 